\title{
Negotiation of Cooperative Maneuvers for Automated Vehicles: Experimental Results
}

\author{
Daniel $\mathrm{He} \beta^{1}$ and Ray Lattarulo ${ }^{2}$ and Joshué Pérez ${ }^{2}$
}

\begin{abstract}
When the number of automated vehicles deployed in public traffic reaches a significant level, their form of interaction with other automated vehicles, human controlled vehicles and non-motorized participants will impact traffic overall safety and efficiency. While many studies have approached impact analysis on the side of efficiency and via simulation, we here elaborate on practically applicable methods for safe cooperation of automated vehicles from the perspective of life-size experimental platforms. The EU project UnCoVerCPS investigates on-line verification based safety analysis and control of cyberphysical system, applying the developed techniques to cooperation of automated vehicles. In a previous publication, a message set for negotiation of such a cooperation has been proposed, (STRP), which is compatible with the investigated verification approach. This publication follows up by providing results and analysis of test drives with two cooperating, automated vehicles with differing software and hardware architectures. The STRP messages are employed to negotiate a cooperative lane change via Car-to-Car radio in an urban scenario with non-trivia road-geometry. The applicability of the distributed cooperation scheme to real-world conditions is demonstrated.
\end{abstract}

\section{INTRODUCTION}

According to some models, automated vehicles should be able to positively impact traffic efficiency [1] and road safety [2]. Consistent and precise longitudinal control as well as small reaction times allow automated vehicles (or even driver assistance systems [3]) to drive higher speeds at smaller inter vehicle distances, thus positively influencing road capacity and stability of the traffic flow. In conflict situations such as ramps, lane merges and un-signaled intersections, traffic efficiency and safety are additionally impacted by the participants' aptness at cooperation. Human drivers are usually highly skilled at (although not always motivated for) implicit cooperation schemes to resolve these situations. Implicit meaning, intentions and agreements are not verbally communicated due to a lack of a communication channel. Instead they are deduced from the observation and extrapolation of driving behavior, e.g. compare [4]. On the one hand, automated vehicles require the capability for implicit cooperation to interact with human traffic participants. On the other hand, it is clear that explicit cooperation, meaning the direct communication of intentions and agreements, increases the performance of cooperations. Assuming some degree of uncertainty in implicit cooperations, a safety conscious AV must behave more conservatively on such a basis than with explicit cooperation agreements. To to utilize precision of

\footnotetext{
${ }^{1}$ Deutsches Zentrum für Luft-und Raumfahrt, Lilienthalpl. 7, 38108 Braunschweig, Germany daniel.hess@dlr.com

${ }^{2}$ Tecnalia Research and Innovation, 48160, Derio, Vizcaya, Spain \{rayalejandro.lattarulo, joshue.perez\}@tecnalia.com
}

control and fast reaction times of $\mathrm{AV}$ for tighter maneuvering in merging, lane changing and intersection scenarios, explicit cooperation approaches should be preferred for interactions between automated vehicles.

The EU project UnCoVerCPS investigates safety of cooperative automated vehicles. Formal verification techniques are employed to analyze actions of the vehicle on-line, during operation [5]. To facilitate the analysis of cooperative actions, a cooperation scheme is required, which leads to unambiguous negotiation states and agreements and supports efficient driving. Employing Vehicle-to-X (V2X) radio to communicate, transmission failures at any point of time during negotiation must be tolerated. Cooperation can be organized by a central controller or vehicles operate as peers in a distributed manner. While central coordination more easily achieves globally optimal vehicle coordinations [6], distributed approaches [7] are wider applicable (i.e. can be used without placing a coordination device at every conflict point) and can be more robust. In the following we discuss a practical approach to distributed negotiation of cooperations, intended to meet above requirements.

The remainder of the paper is organized as follows: Section III describes the state of the art considering cooperative automated vehicles (CAV) and communication protocols. Section III discusses properties of an approach to cooperation of automated vehicles. Section IV presents experimental results for the application of the discussed approach to cooperative lane changing with two automated vehicles IV Finally, our conclusions are shown in section $\mathrm{V}$

\section{STATE OF THE ART}

Several messages for vehicle communication have already been standardized. Yet messages such as ETSI-DENM [8], CAM [9] and others merely support information sharing, without any means for harmonization of intentions. The project MAVEN [10] proposed an extension of the CAmessage in order to carry information for platoon forming and control. In the GCDC i-Game 2016 a Cooperative Lane Change Message or iCLCM was proposed [11]. The message is sent with a frequency between 1 and $25 \mathrm{~Hz}$, this high frequency was assigned to accomplish safety requirements during difficult cooperative maneuvers as platoons merging. The main problem of this approach is its specific designation for the platoon scenario without supporting the general driving process. Moreover, the message payload size is big, with information specific of platoon scenarios: objects, lanes, merge conditions, type of scenario, dynamics of the vehicle (redundant to the CAM), etc. The use of a maneuver 
coordination message (MCM) is proposed in [12] and in the project TransAID [13]. In the first source, the message is employed for distributed negotiation of trajectories for automated vehicles, while in the second case several methods for centralized traffic coordination are added. A message for reserving reserving space-time sets was proposed by the authors in [5], [14].

In [15] a hybrid automaton technique was established for a cooperative platoon scenario. It was divided in two different software architectures, one for platoon and one for normal driving. The events in charge of switching between them were splitting from the platoon and merging to the platoon. In case of splitting, the vehicle generates a new platoon. The problem with this approach was dividing the cooperation in multiple software architectures depending of the state, and the method cannot be directly migrated to other scenarios as overtaking, intersections, etc.

In [16], a hybrid system was proposed. It was tested in the merging scenario. The ego vehicle control had divided dynamics in cruising normally, decelerate while is kept the lane and changing lane conditions. The other participants in the maneuver considered different control dynamics for yielding the lane for the merging, normal merge in the available space and making space available for upcoming merge scenario. Some interesting aspect, like "courtesy", has been introduced and considered but a continuous communication among all participants is demanded and it was limited to a small set possibilities.

In [17] was reviewed some of the methods used for cooperative driving using infrastructure as a link among multiple connected vehicles, unconnected vehicles and the ones operated by humans in terms of mixed traffic. Those methods were strongly based on modifying traffic signals as traffic lights, but this type of approach cannot be used in all circumstances, as for example highways.

In [18] a novel cooperative lane change method was presented. The name of the system was MOBIL (Minimizing Overall Braking Induced by Lane Changes). This system has the goal of minimizing traffic impact and fuel consumption considering lane changes while driving. The approach has considered the impact over the neighbors and the general traffic scenario. Nevertheless, the method was only implemented in simulation and it does not considered a real negotiation among the participants of the maneuver.

\section{APPROACH}

Radio communication enables automated vehicles to exchange their intentions to cooperate in different traffic situations. These intentions can be encoded in many different ways. Furthermore, the procedure of communication, by which agreements over initially conflicting intentions are reached have to be decided. In the following we elaborate on the properties of a space-time reservation procedure (STRP) previously proposed in [14], regarding communication procedure and encoding intentions by reservations.

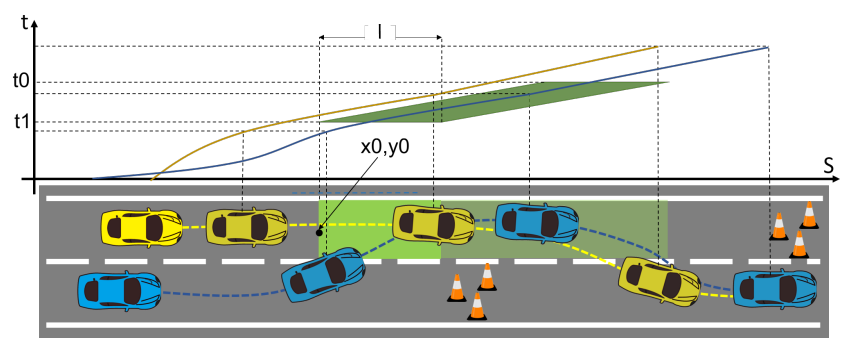

Fig. 1: Lane-based encoding of a reservation for a cooperative lane change

\section{A. Encoding of a reservation}

The topic of vehicle cooperation usually concerns the competition of vehicles for a shared, divisible resource with mutually exclusive access: Space on the road. The allocation of a subset of the resource to a vehicle shall be denoted a reservation. Reservations of space as a function of time could be described in many different ways: Polygons in earth fixed coordinates and time, polygons in lane or road relative, non-linear coordinates, earth fixed or road relative vehicle trajectories [12], buffer spaces attached to the position of certain vehicles [19], positions in a platoon [13], maneuver templates [20] and many more. Previously, we applied lanebased reservations [14], which identify the reserved area by a lane selecting start coordinate $\left(x_{0}, y_{0}\right)$, the extent along the lane $l$, an activation time interval $\left[t_{0}, t_{1}\right]$ and a description of movement along the lane, here based on a constant velocity $v_{0}$. An encoding of such a lane-based reservation is given as a vector:

$$
R_{k}:=\left(t_{0}, t_{1}, x_{0}, y_{0}, l, v_{0}\right)
$$

Fig. 1 gives an example for the reservation on a straight road, with the $s$-coordinate defining a non-linear coordinate system extending in parallel to the lane selected by $\left(x_{0}, y_{0}\right)$. A lane-based reservation has the following properties:

1) The description is efficient and simple, even for complex lane geometries. Varying lane-widths, curved roads and possible obstacles in the lane do not influence the encoding complexity.

2) In contrast to trajectory-based descriptions, a degree of freedom is included in the communicated intentions, which is very natural to the domain. This intended uncertainty allows vehicles to make minor adjustments to their future behavior without necessitating additional negotiations.

3) The description is robust with respect to differing environment perception errors and map data: As long as two cooperating vehicles can agree on the position of a lane with an accuracy of half a lane width, a coordinate intended to be located on the center of a lane uniquely identifies the lane. 4) The explicit formulation of constraints (lane boundaries, position and movement of constraint) is less ambiguous than the exchange of general control strategies or even trajectories, (if the trajectories do not include bounds on maximum deviations due to control errors). 
5) A lane-based reservation can be employed for negotiation of lane changes as well as for negotiation of right of way at an intersection.

6) A downside of the current encoding is that the sharing of a lane at similar longitudinal positions (e.g. for emergency maneuvers) cannot be formulated. Although in general, lanebased solutions for the problem are of course thinkable.

Ultimately, the encoding of the reservation is interchangeable and other forms could be combined with varying negotiation methods and could even be mixed in single or multiple negotiation sessions.

\section{B. Negotiation method}

After deciding, what the subject of a communication is, e.g. here, reservation in a specific encoding, some additional parameters have to be determined: Who is communicating about the subject, what is the timing and order of communication and what are possible results of the communication?

Some approaches require explicit creation of communication groups as a first step [21], [13], other approaches rely on sporadic interactions based on the relevance of the communication topic [12]. The timing of communication can be governed by a fixed rate or triggered by an event. If multiple messages are defined, valid sequences of these messages have to be determined. This encompasses also, whether a negotiation ends after a single or multiple [22] rounds of communication and whether sub-negotiations (compare [12], sec. IV.A.c, “cascading”) are allowed.

The STRP approach foresees communication without predetermined groups in an event-driven manner: Any vehicle, which requires cooperation will spontaneously initiate a negotiation via broadcast of a cooperation request. Any other vehicle, which receives the message, determines whether its cooperation is required. Some simple metrics based on the distance, or more complex metrics based on the road topology can be employed. Next, a required vehicle has to determine its willingness to cooperate, e.g. whether the cost incurred by the cooperation is acceptable. This involves verifying that safety can be maintained under the additional constraints of the requested reservation [5]. Deciding to accept the cooperation, a vehicle has to keep track of the accepted request under all circumstances and signals its guaranteed support via a commit message. For the case that a vehicle decides to reject the cooperation, it will send no reply message at all. Due to the inherent properties of radio communication, the reception of answers can not be guaranteed in any case. Furthermore, due to the dynamics of the system, the relevance of a negotiation rapidly declines and the timeout of the reply of a crucial cooperation partner is assumed to be sufficient indication. A vehicle is not allowed to initiate a sub-negotiation before determining its cooperation with another vehicle. The requesting vehicle can determine from the set of received replies, which vehicle will not interfere with the requested reservation. Additionally employing non-cooperative predictions for all traffic participants, which have not (yet) replied, the requesting vehicle may deduce at any time, whether any other traffic participant could intersect the reserved area - and therefore whether it is safe to enter the reserved area. In the given approach, a successful reservation does not imply the usage of the reserved area. The successfully requesting vehicle may decide against occupying the reservation.

In our approach, the two messages request and commit are defined, which are both sent as a broadcast. The request message specifies the station id of the vehicle initiating the cooperation, $V_{\text {req }} \in \mathbb{N}$, a requested reservation $R_{k}$ and a request identifier $k \in \mathbb{N}$ :

$$
\text { request }:=\left\langle V_{\text {req }}, k, R_{k}\right\rangle
$$

The commit message specifies the three ids $V_{r e q}, k$ and $V_{\text {com }}$, with $V_{\text {com }}$ the id of the vehicle guaranteeing to support the reservation:

$$
\text { commit }:=\left\langle V_{\text {req }}, k, V_{\text {com }}\right\rangle
$$

The proposed negotiation method has the following properties:

1) One requesting and and an arbitrary number of assisting vehicles cooperate spontaneously, triggered by the necessity of the requesting vehicle.

2) For one request only a single round of negotiation is executed and sub-negotiations are prohibited before answering. If a vehicle receives a request, it may first answer with a commit and then in turn send its own request in order to avoid cost incurred by committing to the initial request.

3) Under assumption of arbitrary message delay (loss), the negotiation remains conservatively unambiguous: While the loss of a commit message may lead to unnecessary conservatism (false assumption of the requesting vehicle that a cooperation is not taking place), there is no possible outcome, under which a cooperation is falsely assumed by the requesting vehicle.

4) Non-communicating traffic participants (manually driven vehicles) are naturally accounted for in the negotiation method: As they will not send a commit message, they are handled identically to vehicles whose messages were lost or which are unwilling to cooperate.

5) All non-committing vehicles are predicted according to some basic uncooperative prediction models [23], which allows a requesting vehicle to determine whether a reservation is endangered by non-committing vehicles. Thus a cooperation between automated vehicles may take place in the presence of uncooperative participants, as long as the uncooperative participants do not exhibit adverse behavior.

In the following, a conceptual example is given for a cooperative lane change assisted by multiple vehicles.

\section{Conceptual example}

Cooperation during ramp merging can greatly improve traffic flow. Automated vehicles could theoretically improve on the performance of cooperative human drivers: Precisely specifying cooperation constraints with an approach as such proposed above, misunderstandings are avoided, preparation 


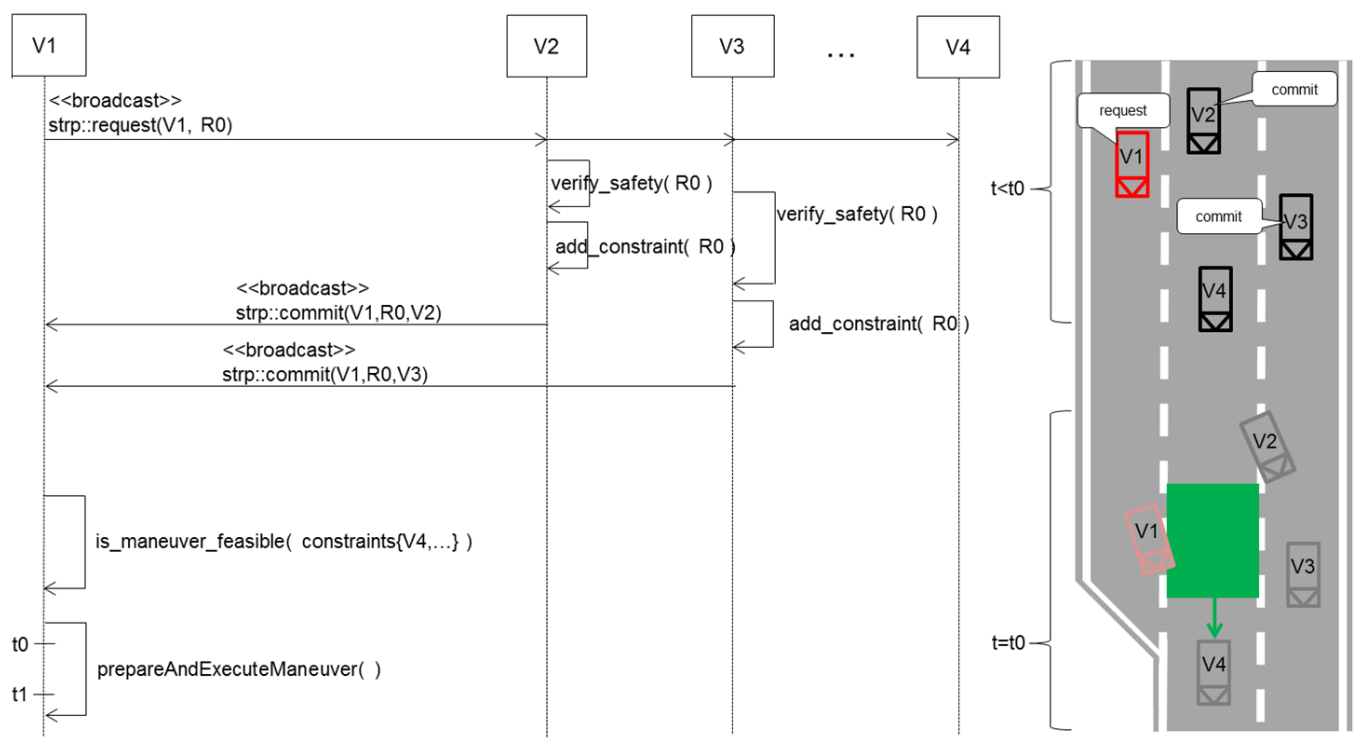

Fig. 2: Negotiation sequence for a cooperative lane change.

time can be increased and inter vehicle distance can be reduced. In order to highlight the application of proposed method to the cooperation of multiple vehicles and the interaction with non-communicating traffic participants, the following example is given: Fig. 2 shows the sequence of messages and the evolution of states for of a lane change executed by V1, assisted by V2 and V3 and ignored by V4: $\mathrm{V} 1$ is located on a ramp and requires assistance in order to enter the center lane before the end of the ramp. According to the rules of the road, vehicles already located on a lane have precedence over vehicles entering the lane. In order to execute a maneuver contradicting the normal precedence rules, V1 requires cooperation. The cooperation is initiated by V1 sending via broadcast a request message containing its own identifier and the description of a desired reservation $R_{0}$, (shown in green). Of the several vehicles in the vicinity, $\mathrm{V} 2$ and V3 receive the message. V4 is representative of all vehicles, which either are not equipped, do not want to cooperate or do not receive the request - usually the difference cannot be determined. On reception of the request, V2 and V3 analyze safety implications and the cost incurred by the reservation. In case of a positive cooperation decision, V2 and V3 add the reservation to the set of safety critical constraints, which governs their maneuver planning. As a next step V2 and V3 each broadcast a commit message, which indicates that they will support reservation $R_{0}$ of $\mathrm{V} 1$. Time progresses, while $\mathrm{V} 1$ receives commit messages from surrounding, equipped vehicles. V1 can at any time determine based on the set of received commit messages and state based predictions of uncooperative vehicles, whether the reserved area will be safe to enter at the time of activation $t_{0}$. In the given example, the non-communicating vehicle V4 continuous at constant speed and thus behaves in such a manner that its future, guaranteed non-intersection with the reservation can be ascertained at times $t \leq t_{0}$. V1 is able to determine feasibility of the cooperative lane change, therefore continues preparation and starts entering the target lane, as soon as all preconditions for the safety of the lane change are satisfied. It should be noted that the actions of vehicles V2 and V3 are not predetermined by the negotiation, as long as the reserved area is not entered.

\section{TEST DRIVES}

The proposed cooperation approach has been realized on two physical, automated vehicles in order to demonstrate the validity of assumptions as well as the applicability of the method in real time, under the influence of measurement errors and disturbances and its robustness towards differing environment models.

\section{A. Experiment Setup}

The cooperation protocol is tested with two automated vehicles. Vehicle 1 (V1) is a Renault Twizy equipped and operated by Tecnalia. Vehicle 2 (V2) is a Volkswagen Passat GTE operated by DLR. Both vehicles use differential GPS for localization. They exchange position, heading and velocity information via ETSI V2X CA-messages at a nominal rate of $10 \mathrm{~Hz}$. The proposed negotiation messages are transmitted in user-defined containers of the CA-message in an eventbased manner with a maximum rate of $10 \mathrm{~Hz}$. Different control approaches and architectures are used, with detailed accounts for V1 given in [24], [25]. V2 applies control software identical to [14] as well as similar hardware equipment. V2 operates a tactical planning module at a rate of $10 \mathrm{~Hz}$ : Each update, 10s long nominal candidate trajectories for lane following and lane changing are generated. One trajectory is selected for execution, depending on its feasibility and the overall goal of minimization of travel time. In case of a cooperation, the trajectory optimization problem is augmented by the additional constraints of the negotiated reservation. The top speed of V1 is set to $6 \mathrm{~m} / \mathrm{s}$ for the scenario and the top speed of V2 is set to $8 \mathrm{~m} / \mathrm{s}$. 


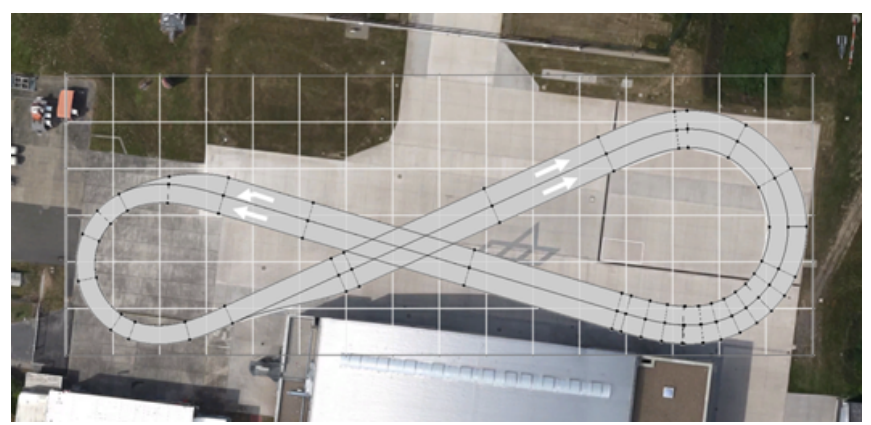

(a) Proving ground with virtual road network

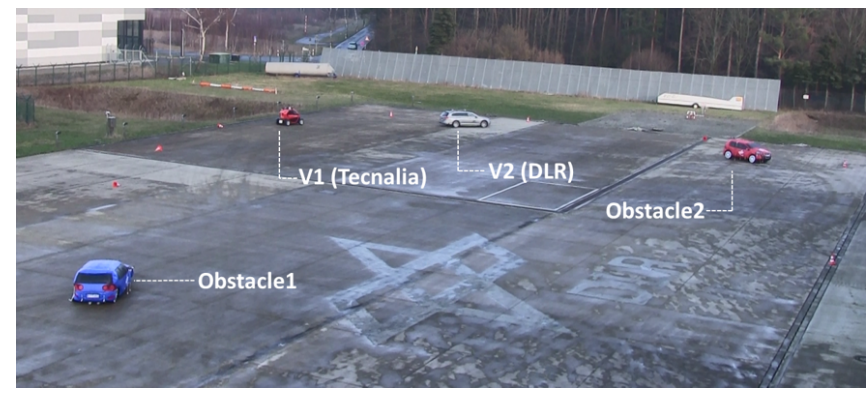

(b) Snapshot of test drive

Fig. 3: Platforms and location of the tests

The test drive is executed on a proving ground at DLR in Braunschweig. Both vehicles load a digital map, shown as an overlay for the (unmarked) proving ground in fig. $3 \mathrm{a}$. In case of V1 the track is loaded from an OpenDrive[26] map ${ }^{1}$ The scenario represents an urban merging situation with a moderately high maximum lane curvature of $0.037 \mathrm{~m}^{-1}$ in the eastern bend and either a single or two parallel lanes of $4 m$ width. A first static obstacle blocks the left lane before the eastern bend and a second static obstacle blocks the right lane at the end of the bend. Vehicles V1 and V2 start in the western part with the slower V1 in front on the single lane and the nominally faster V2 following. An opportunity to change to separate lanes and overtake appears on the ca. $100 \mathrm{~m}$ long section of road between obstacle 1 and 2 . The vehicles negotiate and execute a cooperative lane change on this section. The scenario is repeated 10 times with similar initial conditions induced by the short vehicle following episode at the beginning of each test run.

\section{B. Experiment Results}

The test runs qualitatively similar results. An overview of the course of events of the cooperative lane change is given in fig. 4 for a single test run. The reported times are zeroed at the transmission of the request message, which initiates the cooperation. The situation is depicted starting at $5 s$ before the request with $\mathrm{V} 1$ ahead passing obstacle 1 and ending at $10 \mathrm{~s}$ after the request with $\mathrm{V} 2$ ahead. The aggregate results for the absolute velocity, the longitudinal and the lateral accelerations and the inter-vehicle distance

${ }^{1}$ OpenDrive map download/168401 are given in fig. 5

Both vehicles start off with a velocity of $6 \mathrm{~m} / \mathrm{s}$ and a longitudinal separation of $9 \mathrm{~m}$. V1 moves to the free left lane after passing obstacle 1 . V2 is faced with a typical, distance limited merging situation. V2 accelerates on the right lane and detects that a non-cooperative lane change ahead of $\mathrm{V} 1$ is not feasible. Therefore it sends a request message at $t=0$, which enquires reservation of a subset of the left lane. Depending on the distances towards obstacle 2 and V1, the start of the reservation is requested for a future point of time, on aver age $t=5.8 \mathrm{~s} \mathrm{std}$. dev. $0.1 \mathrm{~s}$, and with a fixed (for this test) duration of $3 \mathrm{~s}$. The reservation associated with the until then unanswered request is shown in red fig. $4 \mathrm{~b}$. The request is received, analyzed for feasibility and answered each test run by $\mathrm{V} 1$ with a commit message. The average reply time was recorded as $0.47 \mathrm{~s}$, see fig. 7

Between $t=0$ and the start of the reservation, V1 brakes with up to $2.5 \mathrm{~m} / \mathrm{s}^{2}$ in order to satisfy the constraints posed by the reservation, reaching a minimum velocity at the start of the reservation. Figs. $4 \mathrm{c}$ to $4 \mathrm{e}$ display the future reserved area in yellow. V2 finishes gap alignment until the start of the reservation, allocating an absolute maximum of ca. $1 \mathrm{~m} / \mathrm{s}^{2}$ longitudinal acceleration. Due to the curvature of the road and the $3 s$ duration of the reservation, the (lateral) acceleration minimizing planner begins the transition to the left lane only ca. $1 s$ after the start of the reservation. V2 completes the lane change until $t=8.5 s$, fig. $4 \mathrm{~g}$ A maximum absolute lateral acceleration of $3.5 \mathrm{~m} / \mathrm{s}^{2}$ is reached directly after completion of the lane change.

\section{CONCLUSION}

This work presents a detailed discussion of the STRP approach to safe cooperation of automated vehicles, previously proposed in [14]. It consists of space-time reservations and the negotiation of this space with the other participants in the immediate area. The approach is applied to cooperative lane changing (merging) and is evaluated with an ETSI CAmessage based implementation of radio communication and two physical test vehicles with different overall architectures and different predictive control approaches. The cooperation approach has several advantageous properties, such as a conservatively unambiguous negotiation outcome, even under assumption of arbitrary communication delays, explicit and unambiguous constraints and low bandwidth requirements due to a simple reservation encoding and a small number of exchanged messages. Our experiments demonstrate the inherent robustness to message delays, real world noisy conditions and differing environment descriptions, as well as the applicability to urban traffic situations. While the cooperation approach excludes un-safe, contradictory negotiation outcomes, conflicting goals are currently only resolved in an implicit "first come, first served" manner, potentially leading to low performance in contentious traffic situations. The extension of the approach towards resolution of conflicting goals will be investigated in future work. 


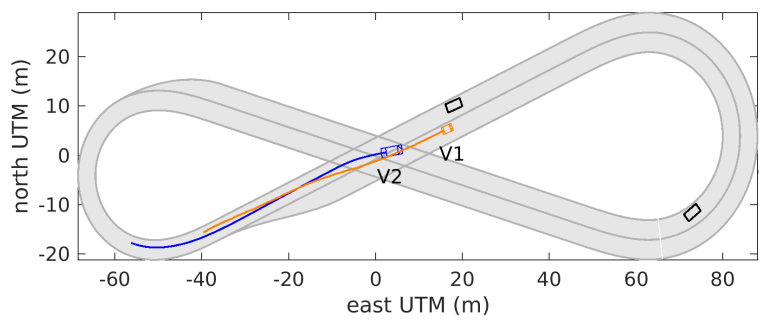

(a) $t=-5 \mathrm{~s}$ before request for cooperation

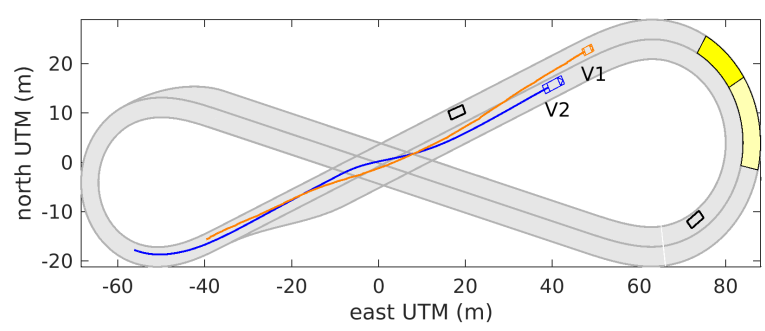

(c) commit received until $t=1 \mathrm{~s}$, cooperation established

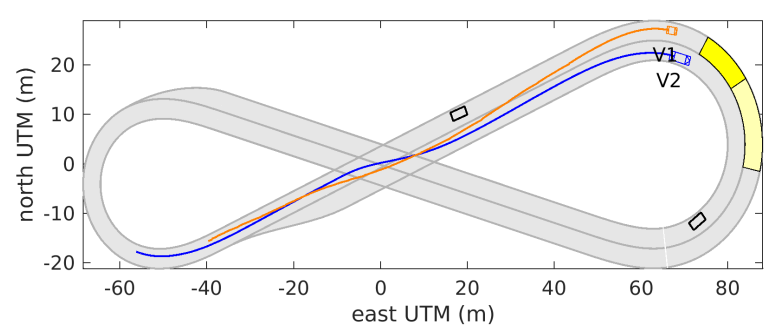

(e) aligning to future reservation area at $t=5 \mathrm{~s}$

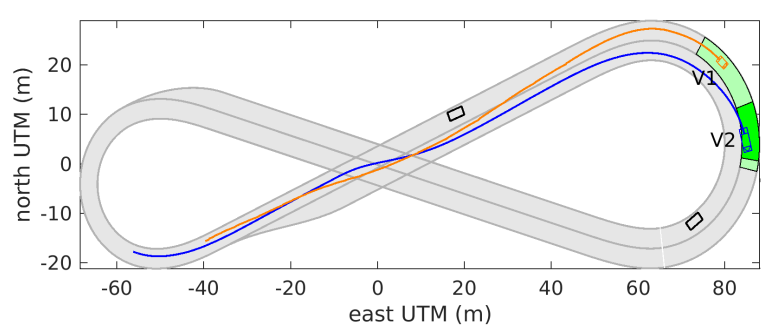

(g) completing coop. lane change at $t=8.5 \mathrm{~s}$

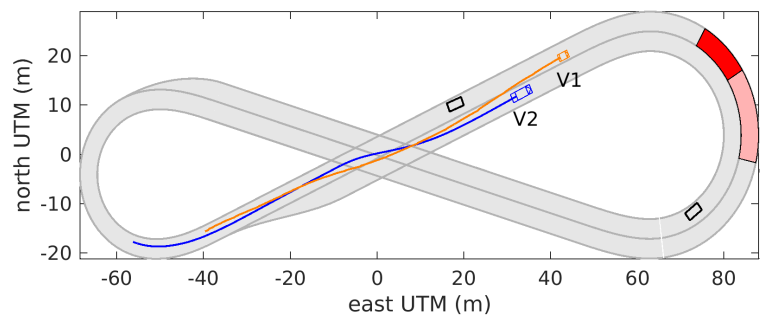

(b) request sent at $t=0 \mathrm{~s}$, waiting for commit

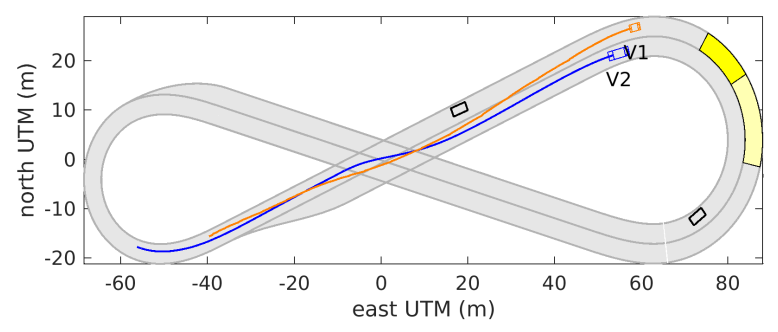

(d) approaching future reservation area at $t=3 \mathrm{~s}$

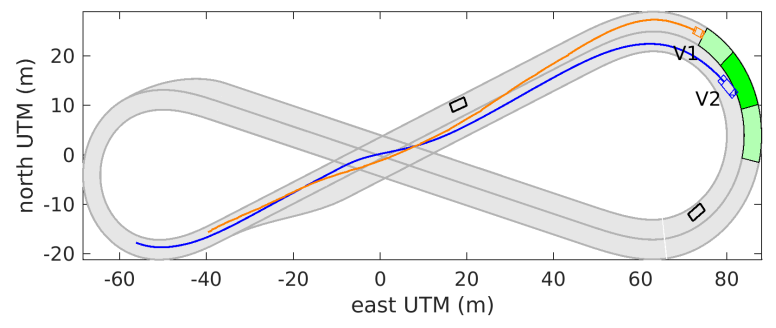

(f) reservation active at $t=7 \mathrm{~s}$ after request

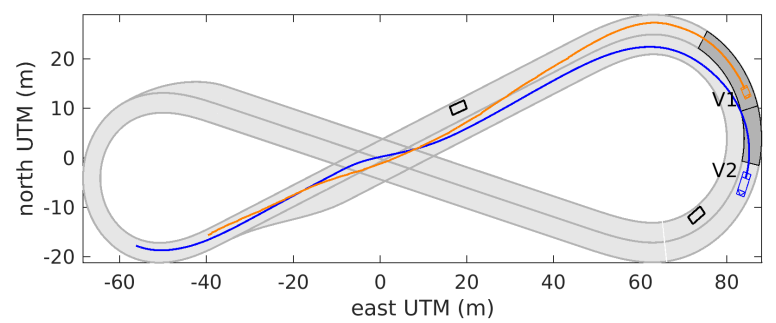

(h) cooperation completed $t=10 \mathrm{~s}$ after request

Fig. 4: Negotiation and execution of a cooperative lane change with two automated vehicles. Test run 6 of 10 .

\section{ACKNOWLEGMENT}

The authors gratefully acknowledge financial support from the European Commission project UnCoVerCPS under grant number 643921 and ECSEL Project ENABLE-S3 with grant agreement number 692455-2.

\section{REFERENCES}

[1] Bernhard Friedrich. The effect of autonomous vehicles on traffic. In Autonomous Driving, pages 317-334. Springer, 2016.

[2] Aso Validi, Thomas Ludwig, Ahmed Hussein, and Cristina OlaverriMonreal. Examining the impact on road safety of different penetration rates of vehicle-to-vehicle communication and adaptive cruise control. IEEE Intelligent Transportation Systems Magazine, 10(4), 2018.

[3] C Lei, EM Van Eenennaam, W Klein Wolterink, G Karagiannis, Geert Heijenk, and J Ploeg. Impact of packet loss on cacc string stability performance. In 2011 11th International Conference on ITS Telecommunications, pages 381-386. IEEE, 2011.
[4] Simon Ulbrich, Simon Grossjohann, Christian Appelt, Kai Homeier, Jens Rieken, and Markus Maurer. Structuring cooperative behavior planning implementations for automated driving. In 2015 IEEE 18th International Conference on Intelligent Transportation Systems, pages 2159-2165. IEEE, 2015.

[5] Daniel Heß, Christian Löper, and Tobias Hesse. Safe cooperation of automated vehicles. AAET Automatisiertes \& Vernetztes Fahren, ITS automotive nord, pages 309-334, 2017.

[6] Jackeline Rios-Torres and Andreas A Malikopoulos. A survey on the coordination of connected and automated vehicles at intersections and merging at highway on-ramps. IEEE Transactions on Intelligent Transportation Systems, 18(5):1066-1077, 2017.

[7] Xiangjun Qian, Jean Gregoire, Arnaud De La Fortelle, and Fabien Moutarde. Decentralized model predictive control for smooth coordination of automated vehicles at intersection. In 2015 European Control Conference (ECC), pages 3452-3458. IEEE, 2015.

[8] ETSI EN 302 637-3 V1.2.2. Intelligent transport systems (its); vehicular communications; basic set of applications; part 3: Specifications 


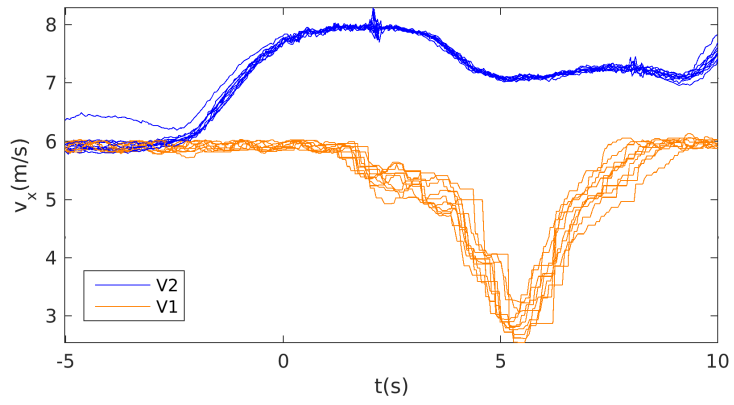

(a) Vehicle speed

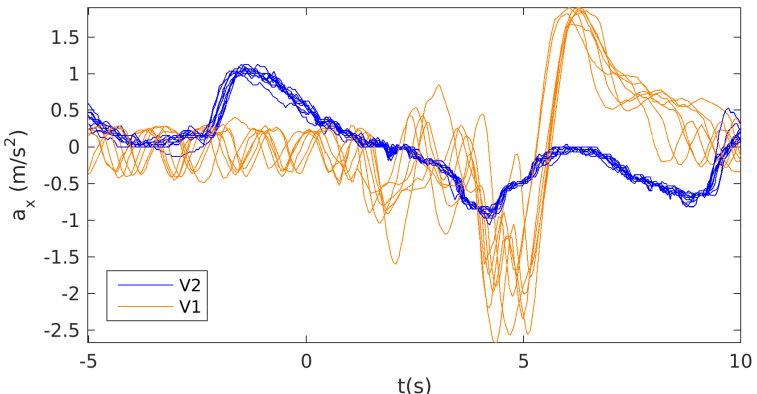

(b) Longitudinal acceleration

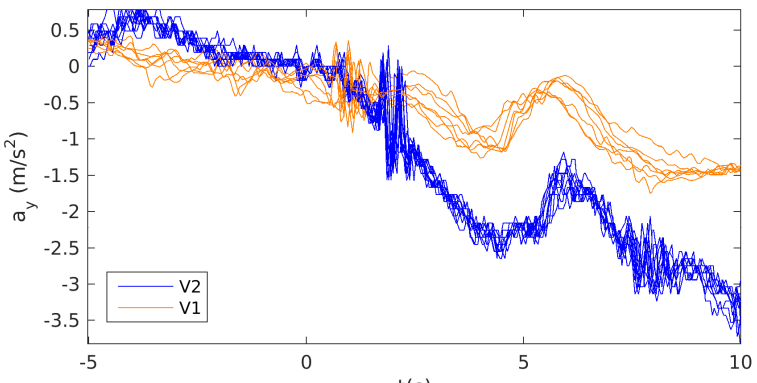

(c) Lateral acceleration

Fig. 5: Speed and acceleration profiles for cooperating vehicles: Time is zeroed at transmission of request message.

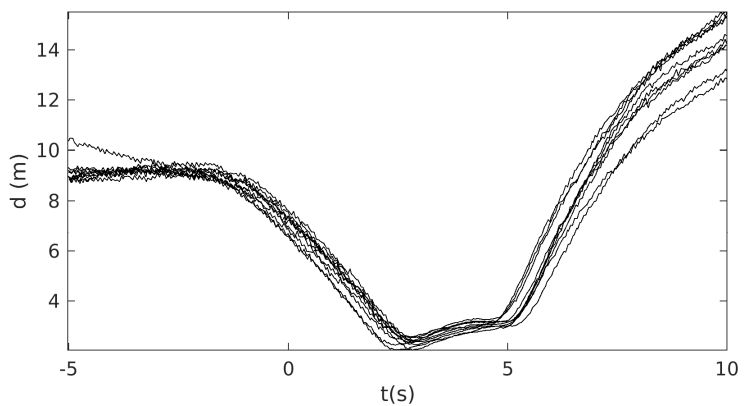

Fig. 6: Minimum distance between cooperating vehicles

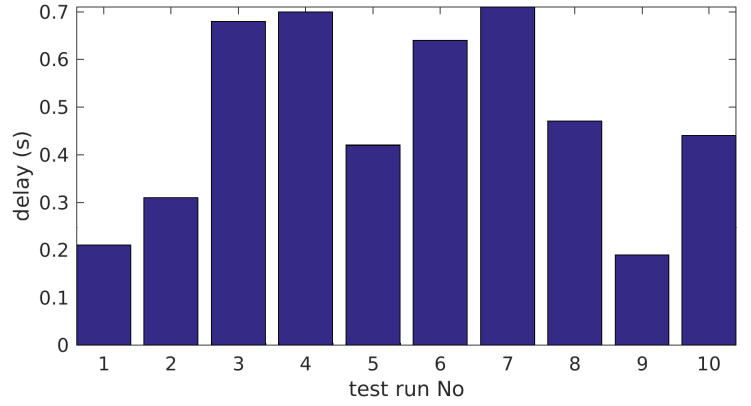

Fig. 7: Delay from request transmission to commit reception

of decentralized environmental notification basic service, 2014.

[9] EN ETSI. 302 637-2 v1. 3.1-intelligent transport systems (its); vehicular communications; basic set of applications; part 2: Specification of cooperative awareness basic service. ETSI, Sept, 2014.

[10] Michele Rondinone, Thomas Walter, Robbin Blokpoel, and Julian Schindler. V2x communications for infrastructure-assisted automated driving. In 2018 IEEE 19th International Symposium on" A World of Wireless, Mobile and Multimedia Networks"(WoWMoM), pages 1419. IEEE, 2018.

[11] Jeroen Ploeg, Elham Semsar-Kazerooni, Alejandro I. Morales Medina, Jan F. C. M. de Jongh, Jacco van de Sluis, Alexey Voronov, Cristofer Englund, Reinder J. Bril, Hrishikesh Salunkhe, Alvaro Arrue, Aitor Ruano, Lorena Garcia-Sol, Ellen van Nunen, and Nathan van de Wouw. Cooperative automated maneuvering at the 2016 grand cooperative driving challenge. IEEE Transactions on Intelligent Transportation Systems, 19(4):1213 - 1226, 2018.

[12] Bernd Lehmann, Hendrik-Jorn Gunther, and Lars Wolf. A generic approach towards maneuver coordination for automated vehicles. IEEE International Conference on Intelligent Transportation Systems (ITSC), pages 3333-3339, 2018.

[13] Julian Schindler, Reza Dariani, Michele Rondinone, and Thomas Walter. Dynamic and flexible platooning in urban areas. In $A A E T$ Automatisiertes und vernetztes Fahren conference 2018, 2018.

[14] Daniel Heß, Ray Lattarulo, Joshue Pérez, Julian Schindler, Tobias Hesse, and Frank Köster. Fast maneuver planning for cooperative automated vehicles. In 21st International Conference on Intelligent Transportation Systems (ITSC), pages 1625-1632. IEEE, 2018.

[15] Zichao Huang, Duanfeng Chu, Chaozhong Wu, and Yi He. Path planning and cooperative control for automated vehicle platoon using hybrid automata. IEEE Transactions on Intelligent Transportation Systems, pages 959 - 974, 2018.

[16] Cristina Menendez-Romero, Mustafa Sezer, Franz Winkler, Christian Dornhege, and Wolfram Burgard. Courtesy behavior for highly automated vehicles on highway interchanges. IEEE Intelligent Vehicles Symposium (IV), pages 943-948, 2018.

[17] Qiangqiang Guoa, Li Li, and Xuegang (Jeff) Ban. Urban traffic signal control with connected and automated vehicles: A survey. Transportation Research Part C: Emerging Technologies, 2019.

[18] Umer Khan, Pavlos Basaras, Lars Schmidt-Thieme, Alexandros Nanopoulos, and Dimitrios Katsaros. Analysing cooperative lane changes models for connected vehicles. IEEE International conference on connected vehicles and expo (ICCVE), pages 565 - 570, 2014

[19] Gregor v. Bochmann, Martin Hilscher, Sven Linker, and Ernst-Rüdiger Olderog. Synthesizing and verifying controllers for multi-lane traffic maneuvers. Formal Aspects of Computing, 29(4):583-600, 2017.

[20] Stefanie Manzinger, Marion Leibold, and Matthias Althoff. Driving strategy selection for cooperative vehicles using maneuver templates. In 2017 IEEE Intelligent Vehicles Symposium (IV), pages 647-654. IEEE, 2017.

[21] Christian Frese, Jurgen Beyerer, and Peter Zimmer. Cooperation of cars and formation of cooperative groups. In 2007 IEEE Intelligent Vehicles Symposium, pages 227-232. IEEE, 2007.

[22] Wei Ren. Consensus based formation control strategies for multivehicle systems. In 2006 American Control Conference, pages 6-pp. IEEE, 2006.

[23] Matthias Althoff, Daniel Heß, and Florian Gambert. Road occupancy prediction of traffic participants. In 16th International IEEE Confer- 
ence on Intelligent Transportation Systems (ITSC 2013), pages 99105. IEEE, 2013.

[24] Ray Lattarulo, Joshue Perez, and Martin Dendaluce. A complete framework for developing and testing automated driving controllers. IFAC-PapersOnLine, pages 258-263, 2017.

[25] Ray Lattarulo, Daniel Heß, and Joshue Perez. A linear model predictive planning approach for overtaking manoeuvres under possible collision circumstances. IEEE Intelligent Vehicles Symposium (IV), pages $1340-1345,2018$.

[26] Marius Dupuis, Martin Strobl, and Hans Grezlikowski. Opendrive 2010 and beyond-status and future of the de facto standard for the description of road networks. In Proc. of the Driving Simulation Conference Europe, pages 231-242, 2010. 